\title{
New Insights on Cosmic Ray Modulation through a Joint Use of Nonstationary Data-Processing Methods
}

\author{
A. Vecchio, ${ }^{1}$ M. Laurenza, ${ }^{2}$ M. Storini, ${ }^{2}$ and V. Carbone ${ }^{1,3}$ \\ ${ }^{1}$ Dipartimento di Fisica, Università della Calabria, 87036 Rende (CS), Italy \\ ${ }^{2}$ IAPS, INAF, Via del Fosso del Cavaliere 100, 00133 Roma, Italy \\ ${ }^{3}$ Liquid Crystal Laboratory, INFM, Ponte P. Bucci Cubo 33B, 87036 Rende (CS), Italy
}

Correspondence should be addressed to A. Vecchio, antonio.vecchio@fis.unical.it

Received 3 August 2012; Accepted 31 October 2012

Academic Editor: Badruddin

Copyright (C) 2012 A. Vecchio et al. This is an open access article distributed under the Creative Commons Attribution License, which permits unrestricted use, distribution, and reproduction in any medium, provided the original work is properly cited.

\begin{abstract}
The time variability of the cosmic ray (CR) intensity, recorded by the Climax neutron monitor and covering the period 1953-2004, has been analyzed by the joint application of the wavelet and the empirical mode decomposition (EMD) analyses. Dominant time scales of variability are found at $\sim 11 \mathrm{yr}, \sim 22 \mathrm{yr}, \sim 6 \mathrm{yr}$ and in the range of the quasi-biennial oscillations (QBOs). The combination of the $11 \mathrm{yr}$ cycle and QBOs explains the Gnevychev Gap (GG) phenomenon and many step-like decreases characterizing the $\mathrm{CR}$ modulation. The additional scales of variability at $\sim 22 \mathrm{yr}$ and $\sim 6 \mathrm{yr}$ are responsible for other features of the long-term $\mathrm{CR}$ trend, such as the intensity flat-topped profile, following the maxima of even-numbered cycles during positive polarity state of the heliosphere $(A>0)$. Comparison with basic time scales of variability derived from the sunspot area (SA) allows the association of the 11 yr cycle and QBOs with solar activity variations, whereas the other two modes with the drift effects govern the CR entrance in the heliosphere.
\end{abstract}

\section{Introduction}

It has been known for a long time that galactic CRs are modulated by solar activity (see the pioneering works performed by Forbush $[1,2])$. In hundred years of research from the CR discovery [3] the topic of cosmic ray modulation has been widely investigated. During the sixties and seventies, the different forms of modulation (short-, medium-, and long-) were identified by a deep analysis of the available experimental data. Solar and interplanetary structures were recognized as the sources of the $3 \mathrm{D}$ variability of the incoming charged particles in the heliosphere and several mathematical techniques were applied on the different datasets during the eighties and nineties to improve the knowledge of the solar-heliospheric domain (see, e.g., [4]). Among them, the Wavelet Transform (WT) was widely recognized as a good tool to investigate the time evolution of periodic or quasi-periodic trends in the experimental data sets $[5,6]$. The WT, by decomposing a time series into the time-frequency space, allows to analyze processes containing multiscale features and it is able to determine both the dominant variability modes and how those modes vary in time. The possibility to evaluate a local spectrum distinguishes the use of the wavelet analysis from, for example, Fourier approaches or other mathematical filtering techniques used in the past. Since this technique provides information both in frequency and time domains it allowed to characterize the evolution of CR variability at different scales $[7,8]$, to identify and characterize the contribution of the different periodicities in the cosmic ray intensity profile for distinct solar cycles and/or different phases of each cycle $[9,10]$, and to correlate variations, detected in solar cycle indices, with the corresponding fluctuations in CR intensity $[11,12]$. Finally the wavelet represented a very useful tool to investigate transitions between different periodicities and to identify a clear difference in the solar activity evolution during odd and even solar activity cycles $[9,10,13,14]$.

More recently, the Empirical Mode Decomposition (EMD) analysis, developed as a signal processing for nonlinear and nonstationary data $[15,16]$, was also introduced in the study of the solar-heliospheric domain (e.g., [17-21]). The method allows to decompose any complicated dataset 
into a finite number of components (called Intrinsic Mode Functions: IMF), representing simple oscillatory modes in the time domain with the signal described by a timedependent amplitude and phase functions.

In this paper we analyze the cosmic ray variations together with sunspot areas recorded for the period from 1953 to 2004. In Section 2 we present results obtained by applying the WT and the EMD to the data set, showing that the EMD modes add more information with respect to the WT only. Section 3 describes two reconstructions of the CR signal, by using different selected EMD modes and discuss the possible association with the different sources of the CR variability, namely solar activity and drift effects. In Section 4 conclusions are drawn.

\section{Data Used and Method of Investigation}

In order to study the behavior of the CR intensity, monthly mean values derived from records of the Climax neutron monitor (Geographic latitude-longitude: N39.37 ${ }^{\circ}$-E253.82 ${ }^{\circ}$; cutoff rigidity: $\sim 3 \mathrm{GV}$; height: $3400 \mathrm{~m}$ a.s.l.; http://ulysses.sr .unh.edu/NeutronMonitor/) have been analyzed for the period 1953-2004. Moreover, monthly averages of sunspot area (SA; http://solarscience.msfc.nasa.gov/greenwch.shtml) have also been considered as a parameter of solar activity responsible for the CR modulation. Search for periodicities in the above data sets is performed by using the WT and the EMD techniques.

As stated in the introduction, the WT analysis offers a significant advantage with respect to the classical Fourier transformation because it allows to localize in time possible periodicities, even those not always present through the whole considered time interval. As mother function we assume the Morlet one, which supplies a better resolution in frequency compared to other available functions. The scales have been chosen as fractional power of 2 :

$$
\begin{gathered}
s_{j}=s_{0} 2^{j \delta_{j}}, \quad \text { for } j=0,1, \ldots, J, \\
J=\delta_{j}^{-1} \log _{2}\left(\frac{N \delta_{t}}{s_{0}}\right) .
\end{gathered}
$$

The following parameters have been used: $\delta_{j}=0.125$, is the frequency resolution, $s_{0}=2$ months, is the smallest resolvable scale, and $J=8 / \delta_{j}$ determines the largest scale. Since the set of scales is nonlinear, the associate error is asymmetric. The left and right uncertainties on each wavelet period $T_{j}$ can be derived as $\Delta T_{j}^{+}=\left(T_{j+1}-T_{j}\right) / 2, \Delta T_{j}^{-}=$ $\left(T_{j}-T_{j-1}\right) / 2$ by using (1) and the relation $T_{j}=1.03 s_{j}$, valid for the Morlet wavelet.

The Wavelet Power Spectrum (WPS), as a function of time and frequency, has been computed in order to characterize the frequencies of the main periodicities. The significance of the power peaks is evaluated against a white noise background [22]. We remark that the wavelet analysis is based on a priori definition of the mother functions, which could not be representative of the eigenfunctions of the investigated phenomenon. For this reason, the EMD, which is an adaptive technique, is more appropriate to deal with nonstationary data [15]. In the EMD framework, a signal $S(t)$ can be decomposed into a finite number $m$ of intrinsic mode functions (IMFs) as

$$
S(t)=\sum_{j=1}^{m} s_{j}(t)+r_{m}(t) .
$$

The mode $s_{j}(t)$ is a zero-mean oscillation, variable in both amplitude and frequency, which can be expressed as $s_{j}(t)=A_{j}(t) \cos \omega_{j}(t)$, where $A_{j}(t)$ and $\omega_{j}(t)$ represent the instantaneous amplitude and phase of the $j$ th EMD mode, respectively. Each IMF has its own characteristic period $\tau_{j}$, defined as the average time difference between $s_{j}(t)$ local extrema (local maxima and minima). The standard deviation of the differences between local extrema quantifies the uncertainty associated with each period. This kind of decomposition is local, complete, and orthogonal [15, 23] and the residue $r_{m}(t)$ in (3) describes the mean trend. The statistical significance of each IMF, with respect to white noise, can be checked by applying a specific test based on the following argument [24]. When EMD is applied to a white noise series, the constancy of the product between the energy density of each IMF and its corresponding averaged period can be deduced. This relation can be used to derive the analytical energy-density spread function of each IMF as a function of different confidence levels. This approach represents the analogy of the statistical significance test used to evaluate the significance levels in the Wavelet power spectrum [22]. Thus, by comparing the energy density of the IMFs extracted from the actual data with the theoretical spread function, IMFs containing information at the selected confidence level can be distinguished from purely noisy modes. Finally, the orthogonality of EMD modes allows to reconstruct the signal at a chosen time scale through partial sums in (3) (see also [17-19]).

We take advantage of both WT and EMD for a detailed study of the CR variability. First, we determine the characteristic periodicities of the signal through the wavelet analysis, which provide frequencies equivalent to the Fourier's ones. Then, we investigate the time variability of all the basic modes, obtained through the EMD, that contribute to the periodicities identified in the WPS. Figure 1 shows the WPS derived from CLI data. The highest values of power are found, as expected, at $T=11.01_{-0.40}^{+0.5} \mathrm{yr}$ and $T=22.04_{-0.90}^{+1.00} \mathrm{yr}$ (where the right and left errors are reported as superscript and subscript, resp.). These periodicities are related to the Schwabe cycle and to the polarity inversion of the Sun's magnetic field (Hale cycle), respectively. Nevertheless, the former cannot be well isolated, because high power is detected in a broad range of periods from 5 to $15 \mathrm{yr}$; the latter is not reliable, being under the cone of influence, determined by the short length of the data set. Enhanced power is also visible between 1 and $4 \mathrm{yr}$, but it is significant only during the maximum phases of cycles 21 and 22, being above the confidence level of $80 \%$ with respect to white noise.

In order to better characterize the oscillations in the frequency ranges identified through the wavelet analysis, we decomposed the CLI data into a set of IMFs. Ten $s_{j}$ modes were obtained and displayed in Figure 2 along with their characteristic periods. The energy density $E_{j}$ of each 


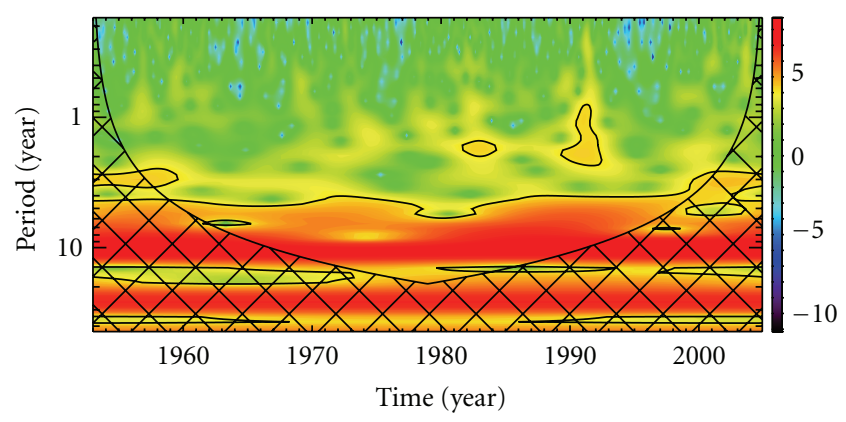

FIGURE 1: WPS of CLI data by using the Morlet mother wavelets. Black contours correspond to the $80 \%$ confidence level. The WPS is meaningless inside the the cone of influence (cross-hatched region). The following parameters have been used: frequency resolution $\delta_{j}=0.125$, Morlet wavelet nondimensional frequency, $w_{0}=6 ; s_{0}=2$ months is the smallest resolvable scale and $J=8 / \delta_{j}$ determines the largest scale.

$\operatorname{IMF}\left(E_{j}=\left\langle s_{j}(t)^{2}\right\rangle\right.$, where $\langle$,$\rangle denotes time averages)$ is shown in Figure 3 as a function of its corresponding characteristic period, to understand which modes dominate the CR variability. The highest amplitude mode $j=8$, having $\tau_{8}=10.6 \pm 0.3 \mathrm{yr}$, can be associated with the Schwabe cycle, whereas $j=9$, having $\tau_{9}=29 \pm 7 \mathrm{yr}$, possibly to the Hale cycle, because its period is comparable with $\sim 22 \mathrm{yr}$ within the error.

The IMF $j=7\left(\tau_{7}=6.5 \pm 0.5 \mathrm{yr}\right)$ is found to be the third important mode, although it was not separated from the $\sim 11 \mathrm{yr}$ periodicity when using the wavelet analysis. Other modes with comparable amplitude are $j=4, j=5, j=6$, having periods $\tau_{4}=1.3 \pm 0.1 \mathrm{yr}, \tau_{5}=2.2 \pm 0.1 \mathrm{yr}, \tau_{6}=$ $3.5 \pm 0.3$, respectively, that is, time scales of the so-called quasi-biennial oscillations (QBOs [25-27]). Mode $j=10$, with $\tau_{10} \sim 41 \mathrm{yr}$, presents only one oscillation which cannot be considered as reliable, given the limited time extent of the data set. Finally, modes $j=1, j=2, j=3$ with $\tau<$ $1 \mathrm{yr}$ have the lowest amplitudes. Moreover, Figure 3 shows that all the computed IMFs are significant, their amplitude being located above the spread lines (dashed, dot-dashed, and dotted lines at 90th, 95th, and 99th confidence level, resp.), obtained by performing the significance test described above.

In order to better understand the links between the identified periodicities and the solar activity, we also performed the EMD analysis on the SA data. The derived modes are shown in Figure 4. Four IMFs $(j=1, j=2, j=3, j=4$, having characteristic periods $\tau_{j}<1$ yr) were found to be not significant at least at 90th confidence level (see Figure 5). Two significant modes $(j=5, j=6)$ have characteristic periods in the QBO range $\left(\tau_{5}=1.9 \pm 0.1 \mathrm{yr}\right.$ and $\tau_{6}=3.5 \pm 0.2 \mathrm{yr}$, resp.), whereas the IMF $j=7$ with $\tau_{7}=10.8 \pm 0.4$ yr has the highest energy (as shown in Figure 5) and it is representative of the Schwabe cycle. Finally, the IMFs $j=8, j=9$ (having periods $\tau_{8}=17.3 \pm 0.6 \mathrm{yr}$ and $\tau_{9}=28.3 \pm 10 \mathrm{yr}$, resp.) could be both related to the shape of $11 \mathrm{yr}$ cycle, possibly related to the Gnevyshev-Ohl rule. We underline that no mode at $\sim 6 \mathrm{yr}$ is detected in SA. This indicates that the IMF $j=7$, detected in CLI data, could not have a solar origin, as will be discussed in Section 3.2.

\section{Reconstruction of the CR Signal}

3.1. CR Modulation Driven by Solar Activity. An important source of CR modulation is represented by solar activity, which is variable on a wide range of temporal scales. The main modulation is at $11 \mathrm{yr}$ time scale and it is related to the well known Schwabe cycle. As a consequence, the CR intensity shows a pronounced $11 \mathrm{yr}$ variation, with the intensity maximum corresponding almost with the solar minimum and vice versa. In addition, many manifestations of solar magnetism as well as some interplanetary phenomena show quasi-biennial variations. The QBOs have been found in photospheric magnetic field and in many solar activity indices such as the sunspot number and area, the number of $H \alpha$ flares [26], the $10.7 \mathrm{~cm}$ radio emission [12], and the coronal emission ([28], and references therein). In the interplanetary medium, QBOs have been observed in the solar wind speed and the interplanetary magnetic field intensity (e.g., [29]), in the number and flux of interplanetary energetic protons at Earth's orbit $[8,27]$, in the density of galactic CRs at $1 \mathrm{AU}[9,14]$, and in the outer heliosphere [10].

It has been shown that the effect of solar activity on CR flux can be essentially described by superposing QBOs to the $\sim 11 \mathrm{yr}$ mode (e.g., $[18,21])$. Thus, in order to investigate the $\mathrm{CR}$ modulation driven by the solar activity we perform the reconstruction of both the $\mathrm{CR}$ and SA signals, by considering QBOs and the $\sim 11 \mathrm{yr}$ mode. As the wavelet analysis indicates that QBOs are located in the Fourier equivalent period $1-4 \mathrm{yr}$, we reconstructed the signal at QBOs scales through partial sums of IMFs as in (3) considering only the significant modes $\left(s_{4}, s_{5}, s_{6}\right)$ having characteristic periods in this range $\left(\tau_{4}=1.3, \tau_{5}=2.2\right.$, $\tau_{6}=3.5$, resp.). This is also consistent with the criterion used by Laurenza et al., [21] for the selection of clear and unambiguous EMD components contributing to the QBOs. Note in Figure 2 that $s_{5}$ is generally the dominant mode for the CLI QBOs, having the highest amplitude during most of the time. Nevertheless, mode $s_{5}$ has a smaller amplitude than $s_{4}$ during 1988-1992, although they are almost in phase with each other. In addition, the $s_{6}$ amplitude is higher only during the period 1953-1967 and comparable with $s_{5}$ during the period 1970-1973. The full QBO signal is depicted in Figure 6 and has a high amplitude during the maximum phase of each solar cycle as expected from results of Bazilevskaya et al. [26], whereas they almost vanish during the sunspot minima.

The importance of QBOs with respect to the $\sim 11 \mathrm{yr}$, around a solar maximum has been evaluated by computing the ratio between the mean square amplitude of the QBOs and that of the $\sim 11 \mathrm{yr}$ mode. Being $s_{\mathrm{QBO}}$ the superposition of the modes $s_{4}, s_{5}, s_{6}$, representing the contribution of the QBO, the ratio $\left\langle\left|s_{\mathrm{QBO}}(t)\right|^{2}\right\rangle /\left\langle\left|s_{8}(t)\right|^{2}\right\rangle$ quantifies the importance of QBOs with respect to the $\sim 11$ yr mode (being the average computed over a time interval of $5 \mathrm{yr}$ around 

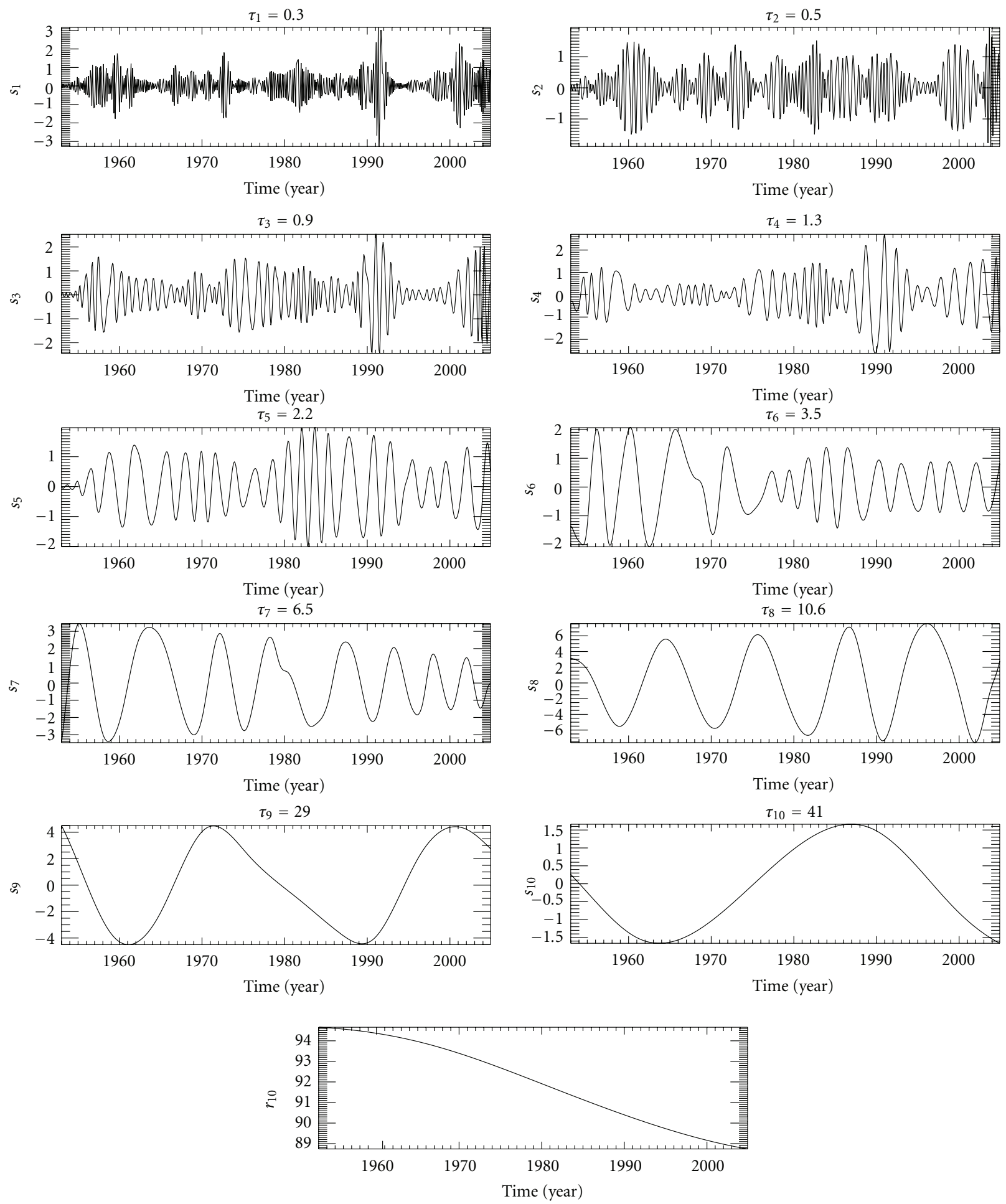

FIGURE 2: IMFs ( $j$ values from 1 to 10 ) obtained by performing the EMD on CLI data, along with their characteristic periods $\tau_{j}$ in years.

each maximum of the $11 \mathrm{yr}$ cycle). The QBO contribution is estimated to be higher than $30 \%$ with respect to the amplitude of the $\sim 11 \mathrm{yr}$ mode for all the considered cycles $(34 \%, 32 \%, 38 \%, 54 \%$, and $31 \%$ for cycles $19,20,21,22$, and 23 , resp., in agreement with the findings of Laurenza et al. [21]).
Then, we compare the superposition of the QBOs and the $\sim 11 \mathrm{yr}$ mode with actual CR data. It is apparent in the upper panel of Figure 7 that the superposition of the $\sim 11 \mathrm{yr}$ and QBOs well reproduces the general trend of CR data, mainly during the maximum and descending phase of sunspot cycles; that is when QBOs reach their highest 


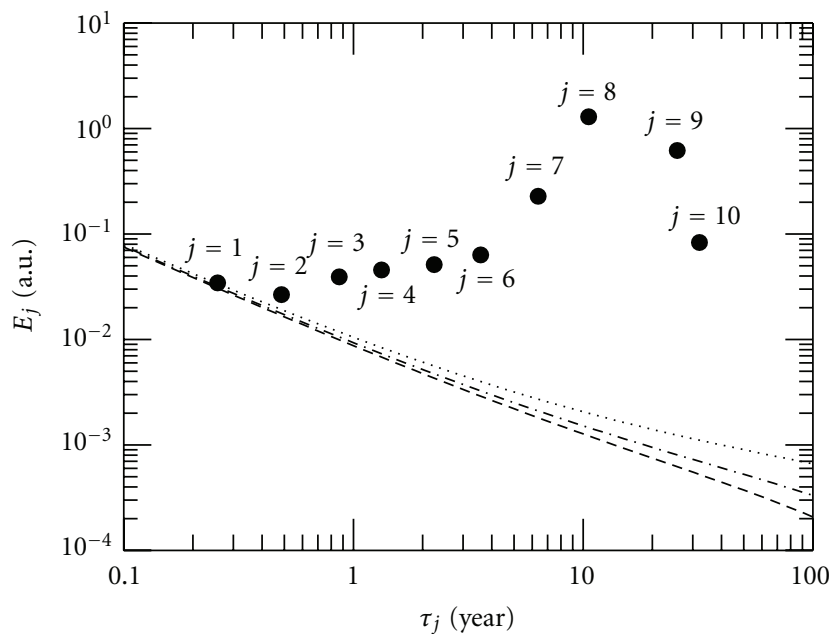

Figure 3: Average amplitude $\left(E_{j}\right)$ versus typical period $\left(\tau_{j}\right)$ of the $j$ IMFs ( $j$ values from 1 to 10 ) identified in the CLI data through the EMD. Dashed, dot-dashed, and dotted lines indicate the 90th, 95th, and 99th percentile, respectively.

amplitude. In particular, this superposition accounts for the majority of the step-like decreases, which characterize the CR modulation. Notice that the so-called mini-cycle during 1974 is reproduced. The cosmic ray depression during the minicycle was related [30] to a decrease in the radial diffusion coefficient $K$ (leading to a reduced efficiency of cosmic ray particles to enter the inner solar system). Under the assumption that $K$ scales inversely with $B$ as $K \sim B^{-2}$, the depression was attributed to increases in the field magnitude through a diffusion dominated process [31], also consistently with findings of Laurenza et al. [21].

The GG phenomenon, namely the peculiar decrease in the temporal trend of various solar-terrestrial indices ([32] and references therein) during the maximum phase of the Schwabe cycles (for early works see $[33,34]$ ), is also observed to be generated by adding QBOs and the $\sim 11 \mathrm{yr}$ mode.

The short term variations of the $\mathrm{CR}$ intensity, not explained by the QBOs, could be due to the so called merged interaction regions [35], acting as a barrier to cosmic rays [36]. In fact, solar wind transient flows may interact with one another and with corotating streams to form a largescale interaction region with a shell-like geometry (global merged interaction region-GMIR) that persists over several solar rotations. These time scales are comparable with the high frequency modes $(\tau<1 \mathrm{yr})$ we detected in CR data, although their possible connection with GMIR has to be further investigated.

We remark that, around sunspot minima, other low frequency modes would be necessary to explain the variability of the CR flux more related to drift effects in the large scale field of the heliosphere that will be treated in the following subsection.

Finally, we study the relationship between medium and long term CR variations and sunspot area. We describe the temporal variability of SA by superposing the $\sim 11 \mathrm{yr}$ mode and QBOs ( $s_{5}$ and $s_{6}$ are the contributing IMFs), which are of the order of $50 \%$ with respect to the $\sim 11 \mathrm{yr}$ component during the maximum phase. Figure 8 shows the reconstruction for both CLI and SA, which present almost the same profile and are shifted between each other. In particular, the GG is well detected in CR for all solar cycles and delayed, with respect to SA, of an average value of about 6 months. Similar results were obtained in the past (e.g., $[37,38])$ in correlative studies between CR data and solar activity indicators. As SA are a proxy for solar activity causing interplanetary disturbances, which act on $\mathrm{CR}$ as propagating diffusion barriers, the found delay can be explained in terms of the propagation time of magnetic perturbations from the Sun towards the edge of CR modulation region. Simple estimations of the propagation time can be obtained for distances of $80 \mathrm{AU}\left(T_{1}\right)$ and $100 \mathrm{AU}\left(T_{2}\right)$, where the cosmic ray modulation should be still important $[39,40]$. In fact, by assuming the average solar wind speed $V^{\mathrm{SW}}=450 \mathrm{~km} / \mathrm{s}$, the $\mathrm{CME}$ mean speed during solar maximum $V^{\mathrm{CME}}=$ $550 \mathrm{~km} / \mathrm{s}$ [41] and the velocity of the high speed streams $V^{\mathrm{HSS}}=750 \mathrm{~km} / \mathrm{s}$ as characteristic velocities of three different solar wind regimes, the corresponding propagation time are $T_{1}^{\mathrm{SW}}=10 \mathrm{months} / T_{2}^{\mathrm{SW}}=13$ months, $T_{1}^{\mathrm{CME}}=8$ months $/ T_{2}^{\mathrm{CME}}=10$ months, $T_{1}^{\mathrm{HSS}}=6$ months $/ T_{2}^{\mathrm{HSS}}=7$ months. Indeed, such values are also consistent with the delay time between transient CR decreases measured at different heliocentric distances, following intense solar-interplanetary events. For instance, a large propagating transient CR decrease was detected at neutron monitor energies at the Earth in October 2003 (the Halloween event; Plainaki et al. [42]) and about 6-8 months later by CR detectors aboard Voyager 2 and Voyager 1 at $73 \mathrm{AU}$ and $92 \mathrm{AU}$, respectively $[43,44]$.

3.2. CR Modulation and Drift Effects. It is widely known that the CR intensity curve follows a $22 \mathrm{yr}$ cycle with alternate maxima being flat-topped and peaked (e.g., [45]). This peculiar behavior is described by models of CR modulation [46-52], based on the observed reversal of the Sun's magnetic field polarity, curvature and gradient drifts in the interplanetary magnetic field. In the drift formalism, during epochs when $A>0$ (i.e., when the Sun's dipole magnetic field is positive in the northern hemisphere) the approach in the inner heliosphere of positively charged CRs is from over the poles, while during epochs when $A<0$ the preferred direction is from along the heliospheric current sheet (HCS). The average excursion, during a solar rotation, of the HCS from the heliographic equator is referred to as tilt angle (e.g., [53]), which characterize the drift effects on CRs (e.g., $[50,54,55])$. In periods of minimum solar activity, that is, when the CR modulation by solar activity is reduced, the effects related to the polarity state of the heliosphere and to the HCS tilt are supposed to be more important. In order to account for the CR behavior described above, modes $s_{7}, s_{9}$ have to be considered. As clearly seen in the lower panel of Figure 7 , the superposition of these IMFs to the $\sim 11 \mathrm{yr}$ one is necessary to reproduce the long term variation of the actual CR data during the minimum solar activity periods and the maxima of odd numbered cycles. Hence, the combination 

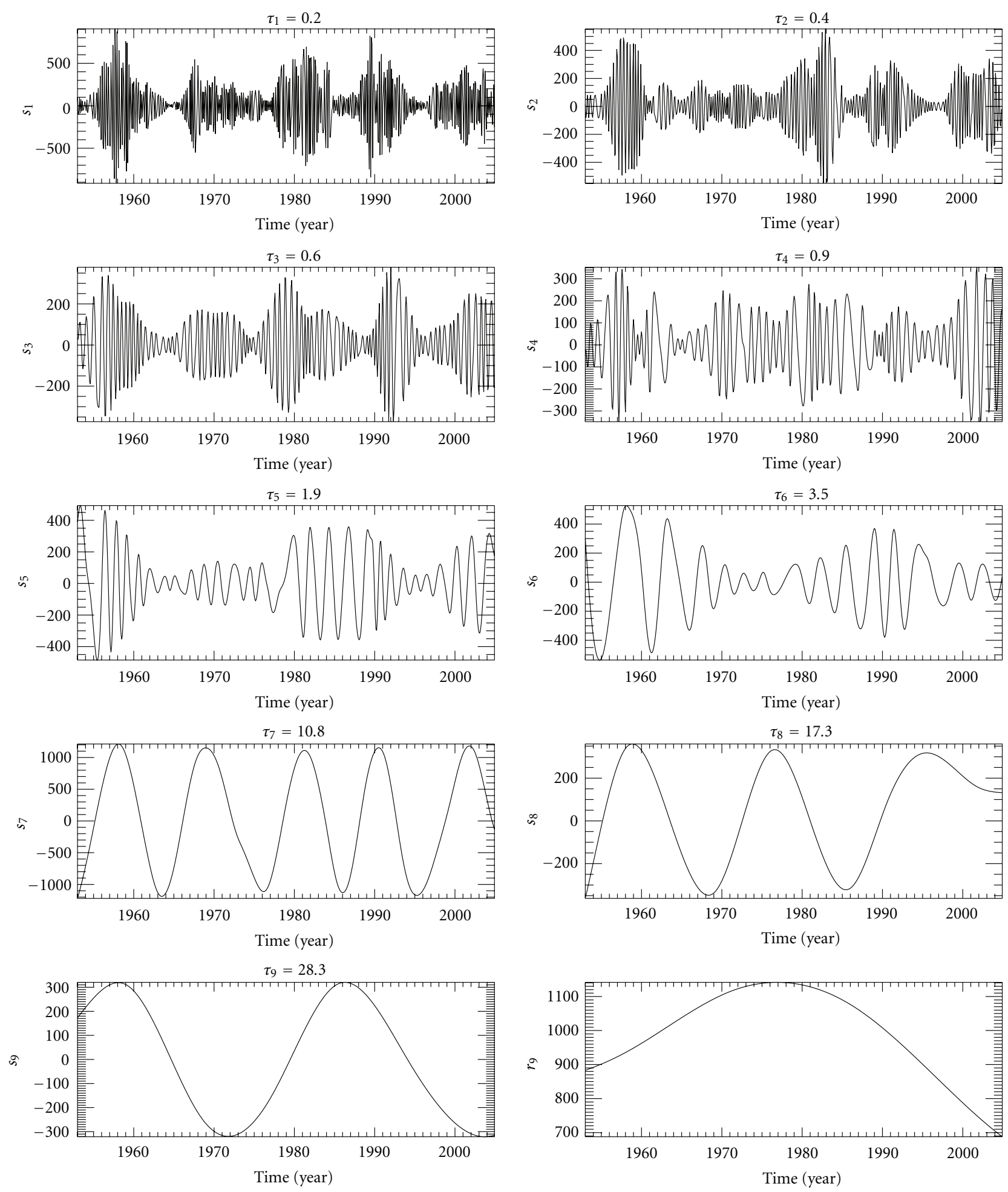

FIgURE 4: IMFs ( $j$ values from 1 to 9 ) obtained by performing the EMD on SA data, along with their characteristic periods $\tau_{j}$ in years.

of both $j=7,9$ IMFs is fundamental to take into account the full contribution of the drifts to the CR modulation. In fact, $s_{9}$ should be representative of the heliosphere polarity changes, its characteristic average period $\tau_{9}$ being $\sim 22 \mathrm{yr}$. In addition, the mode $s_{7}$ seems to be unrelated to solar activity perturbations, because no comparable periodicity was found in the sunspot area, as outlined in Section 2. We suggest that a possible reason for the $\mathrm{CR}$ variability at this time scale can be the particle drift related to the variation during the solar cycle of the latitude extent of the HCS. As a matter of fact, 


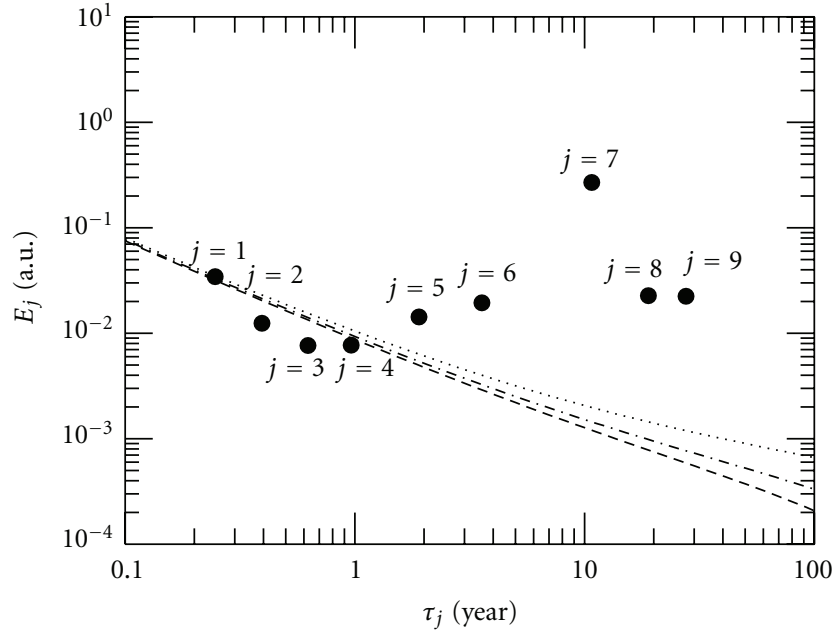

FIGURE 5: Average amplitude $\left(E_{j}\right)$ versus typical period $\left(\tau_{j}\right)$ of the $j$ IMFs ( $j$ values from 1 to 9 ) identified in the SA data through the EMD. Dashed, dot-dashed, and dotted lines indicate the 90th, 95th, and 99th percentile, respectively.

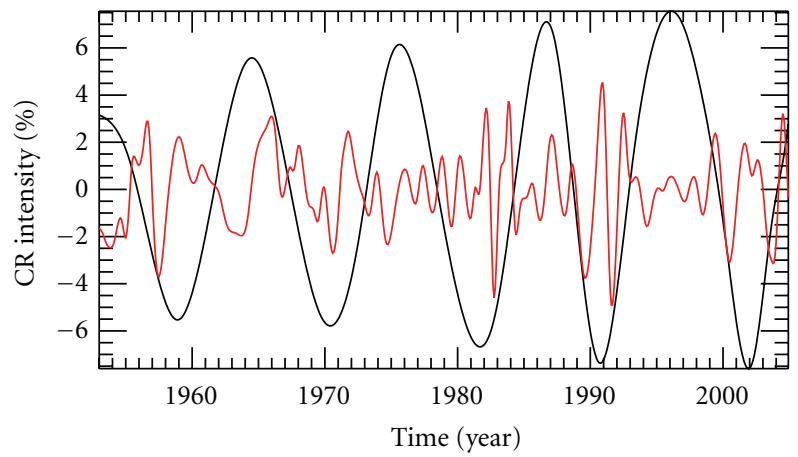

FIgURE 6: Time history of the QBOs (red line) and the $\sim 11 \mathrm{yr}$ mode (black line) obtained through the EMD for CLI data.

the characteristic period $\tau_{7} \sim 6.5 \mathrm{yr}$ is a reasonable average time for the latitudinal excursion of the HCS (although the HCS decrease time during the descending phase of the sunspot cycle is usually higher than the rise time, especially for odd numbered cycles, e.g., [56]). Moreover, the mode $s_{7}$ plays a crucial role in determining the flat-topped maximum of CRs during even sunspot cycles, in agreement with the expectations of drift models that include the changing of HCS inclination during the $11 \mathrm{yr}$ solar cycle (e.g., $[50,55])$. Nevertheless, a better understanding of the nature of the mode $s_{7}$ is needed and will be faced in a future paper.

\section{Conclusions}

A new approach, through the combined application of two powerful techniques, such as the wavelet and EMD, was performed on CR data from the Climax neutron monitor to investigate the time variability and the relationship with the variations of the solar activity, as parametrized by the sunspot area. Our results provide the first evidence for the

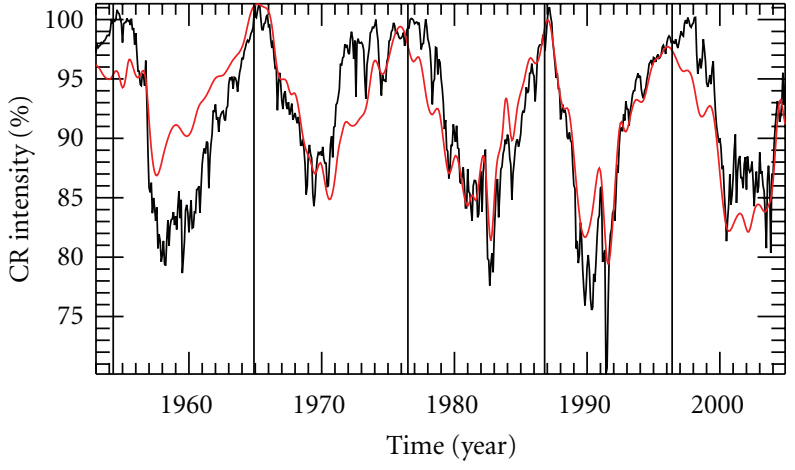

(a)

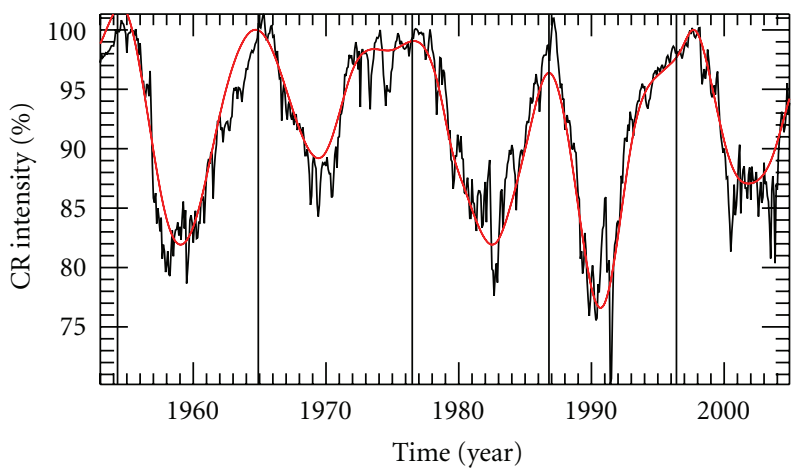

(b)

FIGURE 7: (a) CR intensity (black line), superposition (red line) of the $\sim 11 \mathrm{yr}$ mode, and the QBOs computed from CLI data. (b) CR intensity (black line), superposition (red line) of the $\sim 11 \mathrm{yr}$ and modes $s_{7}$ and $s_{9}$ computed from CLI data. Dashed vertical lines indicate the time of the solar minima as provided by ftp://ftp.ngdc.noaa.gov/STP/SOLAR_DATA/ SUNSPOT_NUMBERS/docs/maxmin.new.

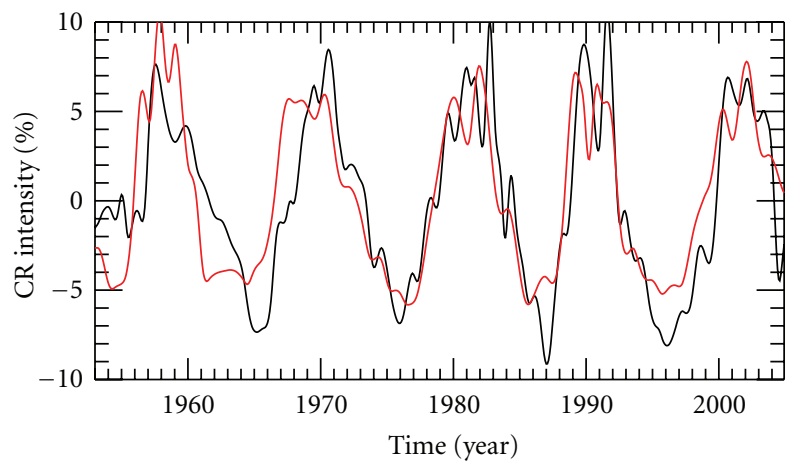

Figure 8: Superposition of the $\sim 11 \mathrm{yr}$ mode and the QBOs computed from CLI (inverted, black line) and SA (red line) data.

different causes producing the CR modulation, identified according to the temporal scales characterizing the CR variability. In particular, we were able to discern the $\mathrm{CR}$ variations directly induced by the solar activity and those possibly related to the particle drift in the large scale magnetic field in the heliosphere. Solar activity is found to be responsible for the GG phenomenon and for most of the 
step-like CR modulation, through the combined effect of the 11 yr cycle and QBOs.

The effect of the drifts has been clearly identified and associated with the $\mathrm{CR}$ variations at $\sim 22 \mathrm{yr}$ and $\sim 6 \mathrm{yr}$. In particular, time variations at $\sim 22 \mathrm{yr}$ are due to the polarity state of the heliosphere, whereas those at $\sim 6 \mathrm{yr}$ could reflect the latitudinal excursion of the HCS. Both contributions explain the CR time profile, as expected from the drift theory and models, during the descending and minimum phase of solar activity of even numbered solar cycles (compare top and bottom panel of Figure 7), that is when $A>0$ and the CRs enter the heliosphere from the poles, while the HCS tilt decays rapidly to low angles, leading to a fast recovery of the CR intensity soon after the solar maximum. In addition, the shape of the CR intensity during the ascending and maximum phase of the odd numbered cycles (again in periods when $A>0$ ) is determined by these variations as well.

\section{Acknowledgments}

This work was partially supported by the ASI/INAF Contract no. I/022/10/0, by the European Social Fund, European Commission, and by Regione Calabria. Thanks are due also to the Italian PNRA for the use of the RAC-ANT database, prepared at the former IFSI-Roma (now IAPS) under Contract PROGDEF09_37 (code 2009/A3.07).

\section{References}

[1] S. E. Forbush, "World-wide cosmic ray variations, 1937-1952," Journal of Geophysical Research, vol. 59, p. 525, 1954.

[2] S. E. Forbush, "Time variations of cosmic rays," in Handbuch der Physik, S. Flugge, Ed., vol. 49, p. 159, Springer, New York, NY, USA, 1966.

[3] V. F. Hess, Zeitschrift Für Physik, vol. 13, p. 1084, 1912.

[4] M. Storini, "Cosmic rays for solar-terrestrial physics," Nuovo Cimento della Societa Italiana di Fisica C, vol. 20, no. 6, pp. 871-880, 1997.

[5] I. Dauberchies, "The wavelet transform, time-frequency localization and signal analysis ," IEEE Transactions on Information Theory, vol. 36, pp. 961-1005, 1990.

[6] P. Kumar and E. Foufoula-Georgiou, "Wavelet analysis for geophysical applications," Reviews of Geophysics, vol. 35, no. 4, pp. 385-412, 1997.

[7] K. Kudela, S. Yasue, K. Munakata, and P. Bobik, "cosmicray variability at different scales: a wavelet approach," in Proceedings of the 26th International Cosmicray Conference, vol. 7, pp. 163-166, Salt Lake City, Utah, USA, August 1999.

[8] M. Laurenza, S. Giangravé, M. Storini et al., in Proceedings of the 30th International Cosmicray Conference. July 3-11, 2007, Mrida, Yucatn, Mexico, R. Caballero, J. C. D’Olivo, G. MedinaTanco, L. Nellen, F. A. Snchez, and J. F. Valds-Galicia, Eds., vol. 1, pp. 513-516, Universidad Nacional Autónoma de México, Mexico City, Mexico, 2008.

[9] K. Kudela, J. Rybak, A. Antalovà, and M. Storini, "Time evolution of low-frequency periodicities in cosmic ray intensity," Solar Physics, vol. 205, pp. 165-175, 2002.

[10] C. Kato, K. Munakata, S. Yasue, K. Inoue, and F. B. McDonald, "A $\sim 1.7$-year quasi-periodicity in cosmic ray intensity variation observed in the outer heliosphere," Journal of Geophysical Research A, vol. 108, no. 10, Article ID 1367, 7 pages, 2003.

[11] J. Rybàk, A. Antalovà, and M. Storini, "The wavelet analysis of the solar and cosmic-ray data," Space Science Reviews, vol. 97, no. 1-4, pp. 359-362, 2001.

[12] J. F. Valdés-Galicia and V. M. Velasco, "Variations of mid-term periodicities in solar activity physical phenomena," Advances in Space Research, vol. 41, pp. 297-305, 2008.

[13] M. Laurenza and M. Storini, Memorie Della Societa Astronomica Italiana, vol. 9, p. 109, 2006.

[14] M. Laurenza and M. Storini, in Proceedings of the 31st International Cosmicray Conference, 2009, paper ID: 00475, http://icrc2009.uni.lodz.pl..

[15] N. E. Huang, Z. Shen, S. R. Long et al., "The empirical mode decomposition and the Hilbert spectrum for nonlinear and non-stationary time series analysis," Royal Society of London Proceedings A, vol. 454, p. 903, 1998.

[16] P. Flandrin, G. Rilling, and P. Gonçalvés, "Empirical mode decomposition as a filter bank," IEEE Signal Processing Letters, vol. 11, no. 2, pp. 112-114, 2004.

[17] J. Terradas, R. Oliver, and J. L. Ballester, "Application of statistical techniques to the analysis of solar coronal oscillations," Astrophysical Journal, vol. 614, no. 1, pp. 435-447, 2004.

[18] A. Vecchio, M. Laurenza, V. Carbone, and M. Storini, "Quasibiennial modulation of solar neutrino flux and solar and galactic cosmic rays by solar cyclic activity," Astrophysical Journal Letters, vol. 709, no. 1, pp. L1-L5, 2010.

[19] A. Vecchio, M. Laurenza, D. Meduri, V. Carbone, and M. Storini, "The dynamics of the solar magnetic field: polarity reversals, butterfly diagram, and quasi-biennial oscillations," Astrophysical Journal, vol. 749, no. 1, Article ID 27, 2012.

[20] B. L. Barnhart and W. E. Eichinger, "Analysis of sunspot variability using the hilbert-huang transform," Solar Physics, vol. 269, no. 2, pp. 439-449, 2011.

[21] M. Laurenza, A. Vecchio, V. Carbone, and M. Storini, "Quasibiennial modulation of galactic cosmic rays," The Astrophysical Journal, vol. 749, p. 167, 2012.

[22] C. Torrence and G. P. Compo, "A practical guide to wavelet analysis," Bulletin of the American Meteorological Society, vol. 79, no. 1, pp. 61-78, 1998.

[23] D. A. T. Cummings, R. A. Irizarry, N. E. Huang et al., "Travelling waves in the occurrence of dengue haemorrhagic fever in Thailand," Nature, vol. 427, no. 6972, pp. 344-347, 2004.

[24] Z. Wu and N. E. Huang, "A study of the characteristics of white noise using the empirical mode decomposition method," Proceedings of the Royal Society A, vol. 460, no. 2046, pp. 15971611, 2004.

[25] E. E. Benevolenskaya, "A model of the double magnetic cycle of the sun," Astrophysical Journal Letters, vol. 509, no. 1, pp. L49-L52, 1998.

[26] G. A. Bazilevskaya, M. B. Krainev, V. S. Makhmutov, E. O. Flückiger, A. I. Sladkova, and M. Storini, "Structure of the maximum phase of solar cycles 21 and 22," Solar Physics, vol. 197, no. 1, pp. 157-174, 2000.

[27] G. A. Bazilevskaya, V. S. Makhmutov, and A. I. Sladkova, "Gnevyshev gap effects in solar energetic particle activity," Advances in Space Research, vol. 38, no. 3, pp. 484-488, 2006.

[28] A. Vecchio and V. Carbone, "Spatio-temporal analysis of solar activity: main periodicities and period length variations," Astronomy and Astrophysics, vol. 502, no. 3, pp. 981-987, 2009.

[29] E. Forgàcs-Dajka and T. Borkovits, "Searching for mid-term variations in different aspects of solar activity-looking for probable common origins and studying temporal variations of 
magnetic polarities," Monthly Notices of the Royal Astronomical Society, vol. 374, pp. 282-291, 2007.

[30] G. Wibberenz and H. V. Cane, "Simple analytical solutions for propagating diffusive barriers and application to the 1974 minicycle," Journal of Geophysical Research, vol. 105, no. 8, p. 18315, 2000.

[31] G. Wibberenz, H. V. Cane, I. G. Richardson, and T. T. von Rosevinge, "The influence of tilt angle and magnetic field variations on cosmic ray modulation," Space Science Reviews, vol. 97, pp. 343-347, 2001.

[32] M. Storini, G. A. Bazilevskaya, E. O. Flückiger, M. B. Krainev, V. S. Makhmutov, and A. I. Sladkova, "The Gnevyshev gap: a review for space weather," Advances in Space Research, vol. 31, no. 4, pp. 895-900, 2003.

[33] M. Storini, "Testing solar activity features during the descending phase of sunspot cycle 22," Advances in Space Research, vol. 16, no. 9, pp. 51-55, 1995.

[34] F. Feminella and M. Storini, "Large-scale dynamical phenomena during solar activity cycles," Astronomy and Astrophysics, vol. 322, no. 1, pp. 311-319, 1997.

[35] L. F. Burlaga, F. B. McDonald, and N. F. Ness, "Cosmic ray modulation and the distant heliospheric magnetic field: voyager 1 and 2 observations from 1986 to 1989," Journal of Geophysical Research, vol. 98, no. 1, p. 1, 1993.

[36] M. S. Potgieter and J. A. le Roux, "The long-term heliospheric modulation of galactic cosmic rays according to a timedependent drift model with merged interaction regions," The Astrophysical Journal, vol. 423, p. 817, 1994.

[37] I. Sabbah and M. Rybanský, "Galactic cosmic ray modulation during the last five solar cycles," Journal of Geophysical Research A, vol. 111, Article ID A01105, 2006.

[38] M. Singh, Y. P. Singh, and Badruddin, "Solar modulation of galactic cosmic rays during the last five solar cycles," Journal of Atmospheric and Solar-Terrestrial Physics, vol. 70, pp. 169-183, 2008.

[39] W. R. Webber, "Voyager measurements of galactic cosmic rays and implications for modulation in the heliosheath and beyond," in Proceedings of the 5th Annual International Astrophysics Conference, vol. 858, pp. 135-140, March 2006.

[40] M. S. Potgieter, "Challenges to cosmic ray modeling: from beyond the solar wind termination shock," Advances in Space Research, vol. 41, pp. 245-458, 2008.

[41] N. Gopalswamy, S. Nunes, S. Yashiro, and R. A. Howard, "Variability of solar eruptions during cycle 23," Advances in Space Research, vol. 34, no. 2, pp. 391-396, 2004.

[42] C. Plainaki, A. Belov, E. Eroshenko, V. Kurt, H. Mavromichalaki, and V. Yanke, "Unexpected burst of solar activity recorded by neutron monitors during October-November 2003," Advances in Space Research, vol. 35, no. 4, pp. 691-696, 2005.

[43] J. D. Richardson, C. Wang, J. C. Kasper, and Y. Liu, "Propagation of the October/November 2003 CMEs through the heliosphere," Geophysical Research Letters, vol. 32, Article ID L03S03, 4 pages, 2005.

[44] D. S. Intriligator, W. Sun, M. Dryer, C. D. Fry, C. Deehr, and J. Intriligator, "From the Sun to the outer heliosphere: Modeling and analyses of the interplanetary propagation of the October/November (Halloween) 2003 solar events," Journal of Geophysical Research A, vol. 110, no. 9, Article ID A09S10, 2005.

[45] E. J. Smith, Journal of Geophysical Research, vol. 95, p. 731, 1990.
[46] J. R. Jokipii, E. H. Levy, and W. B. Hubbard, "Effects of particle drift on cosmic-ray transport. I-general properties, application to solar modulation," Astrophysical Journal Letters, vol. 213, pp. 861-868, 1977.

[47] M. S. Potgieter and J. A. le Roux, "The simulated features of heliospheric cosmic-ray modulation with a time-dependent drift model. I-general effects of the changing neutral sheet over the period 1985-1990," The Astrophysical Journal, vol. 386, pp. 336-346, 1992.

[48] J. A. le Roux and M. S. Potgieter, "The simulated features of heliospheric cosmic-ray modulation with a time-dependent drift model. II - on the energy dependence of the onset of new modulation in 1987," The Astrophysical Journal, vol. 390, pp. 661-667, 1992.

[49] J. A. le Roux and M. S. Potgieter, "The simulated features of heliospheric cosmic-ray modulation with a time-dependent drift model. IV-the role of heliospheric neutral sheet deformation," The Astrophysical Journal, vol. 397, pp. 686-693, 1992.

[50] S. E. S. Ferreira and M. S. Potgieter, Advances in Space Research, vol. 32, p. 2003, 2004.

[51] S. E. S. Ferreira and M. S. Potgieter, "Long-term cosmic-ray modulation in the heliosphere," The Astrophysical Journal, vol. 603, p. 744, 2004.

[52] R. Manuel, S. E. S. Ferreira, and M. S. Potgieter, "Cosmic ray modulation in the outer heliosphere: predictions for cosmic ray intensities up to the heliopause along Voyager 1 and 2 trajectories," Advances in Space Research, vol. 48, pp. 874-883, 2011.

[53] J. T. Hoeksema, J. M. Wilcox, and P. H. Scherrer, "Structure of the heliospheric current sheet in the early portion of Sunspot Cycle 21," Journal of Geophysical Research, vol. 87, p. 10331, 1982.

[54] J. R. Jokipii and B. Thomas, "Effects of drift on the transport of cosmic rays. IV-modulation by a wavy interplanetary current sheet," Astrophysical Journal Letters, vol. 243, pp. 1115-1122, 1981.

[55] K. Kota and J. R. Jokipii, "Effects of drift on the transport of cosmic rays. VI- a three-dimensional model including diffusion," The Astrophysical Journal, vol. 265, pp. 573-581, 1983.

[56] E. W. Cliver and A. G. Ling, "22 Year patterns in the relationship of sunspot number and tilt angle to cosmic-ray intensity," Astrophysical Journal Letters, vol. 551, no. 2, pp. L189-L192, 2001. 

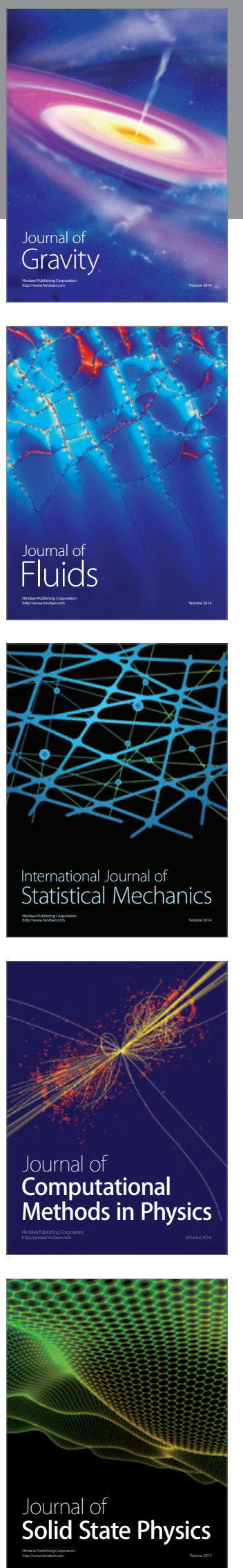

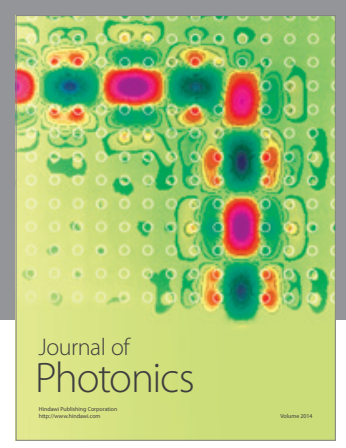

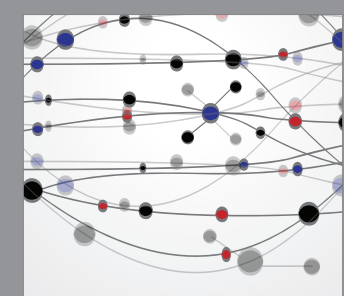

The Scientific World Journal
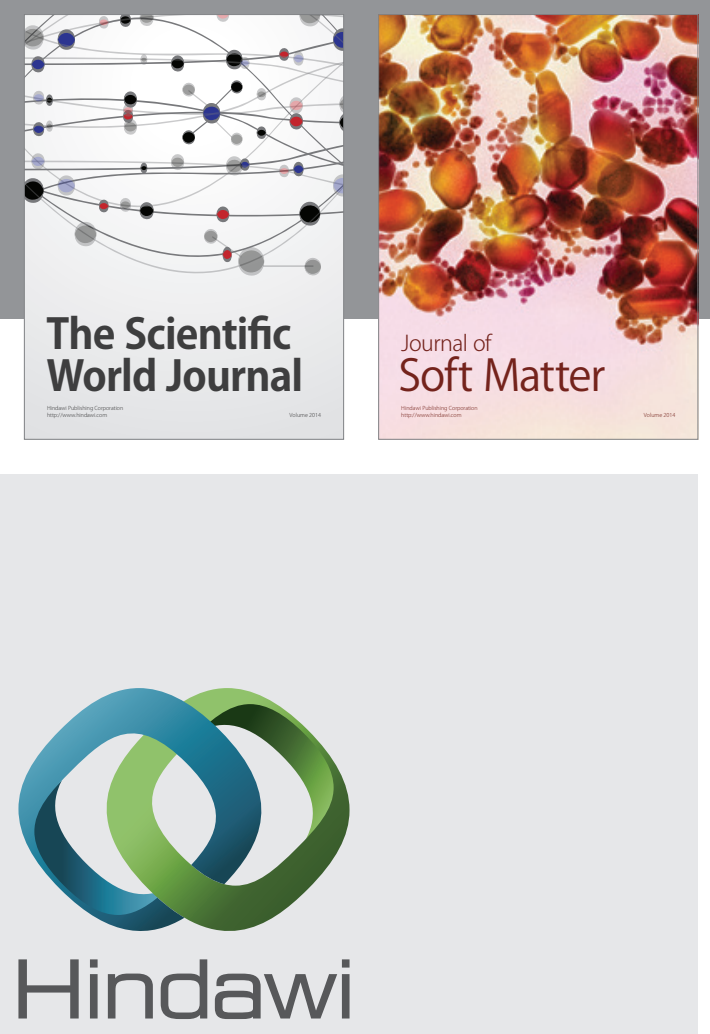

Submit your manuscripts at

http://www.hindawi.com
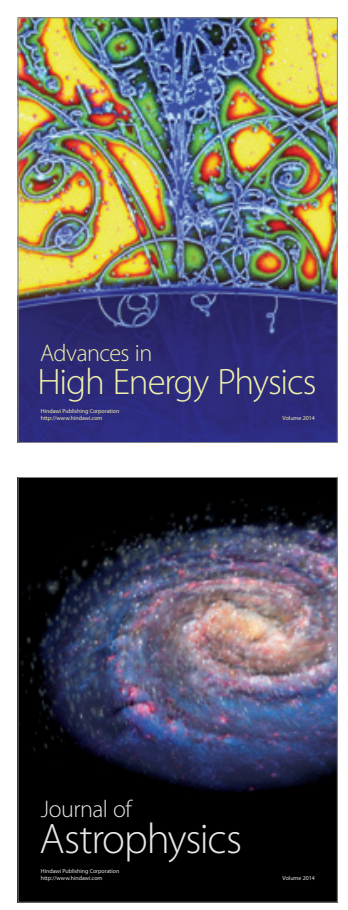
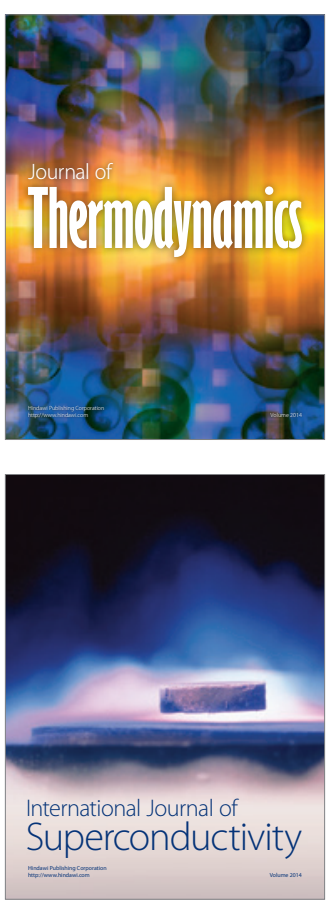
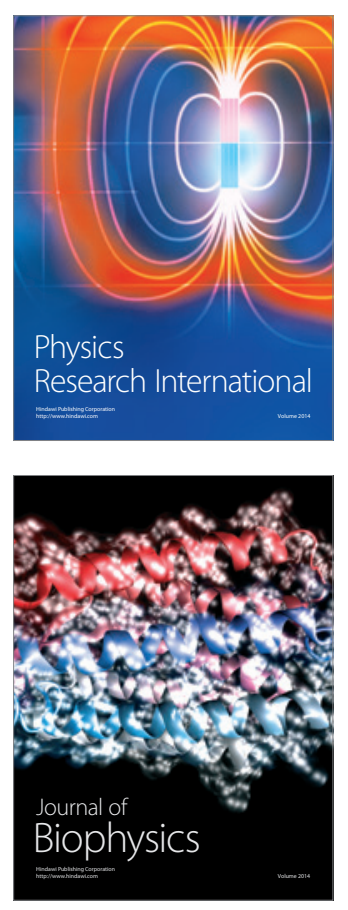
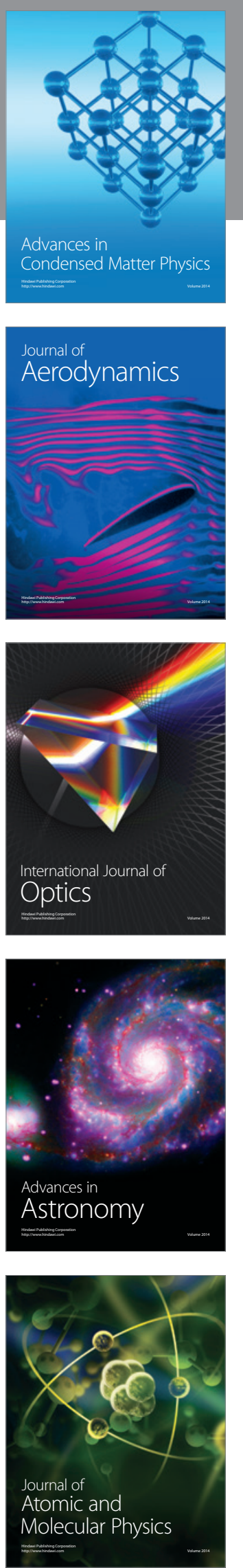